\title{
Identification and Characterization of a Potential Antibiotic Producing Strain of Pantoea ananatis
}

\author{
Michael J. LaGier ${ }^{\bowtie}$, Mark McDaniel, Alyssa Ragner, Amber Castillo \\ Department of Biology, Grand View University, Des Moines, IA, USA. \\ $\triangle$ Corresponding author: Michael J. LaGier, Department of Biology, Grand View University, 1200 Grand View Avenue, Des Moines, IA 50316, USA. Phone: 00 \\ +1515-263-2873; E-mail: mlagier@grandview.edu. \\ (C) The author(s). This is an open access article distributed under the terms of the Creative Commons Attribution License (https://creativecommons.org/licenses/by/4.0/). \\ See http://ivyspring.com/terms for full terms and conditions.
}

Received: 2021.12.14; Accepted: 2022.01.24; Published: 2022.02.07

\begin{abstract}
Antibiotic resistance continues to be a significant public health challenge. Soil bacteria represent a potential source of yet to be discovered antimicrobials. The screening of lowa (United States) soils yielded the identification of a strain of Pantoea ananatis (MMB-1), which displayed an antimicrobialproducing phenotype against a bacterium (Bacillus subtilis) representative of Gram-positive bacteria. Crude, organic, extracts of MMB-1 retained the anti-microbial activity. The draft genome of strain MMB- 1 contains a total of 4,634,340 bp, and 4,624 protein-encoding genes. Consistent with phenotypic observation, the genome of MMB-1 encodes for a number of putative secondary metabolite biosynthetic gene clusters, including those known to be involved in the production of the antibiotics lankacidin $C$ and bottromycin. This study increases our overall understanding of Panteoa as a group, and is also consistent with the notion that members of this genus have significant potential as useful natural product producers.
\end{abstract}

Key words: Pantoea, antibiotic, soil

\section{Introduction}

Members of the Genus Pantoea include at least 35 known species (http://www.bacterio.net), which can be found in a variety of habitats, both terrestrial and aquatic [1]. Pantoea are Gram-negative bacteria, rod-shaped, and often produce mucoid colonies when grown on semi-solid media [1]. A number of identified species produce antimicrobials, and have been developed into biocontrol products, including Bloomtime Biological, which is used to control apple and pear fire blight [2]. Others are thought to contain bioremediation potential, specifically, in the degradation of herbicides [3]. Some species of this genus also appear to behave as plant and animal pathogens [1].

The goal of the current study was to screen Iowa soils, close to the Des Moines metropolitan area, for soil bacteria that show the potential to produce antimicrobial substances. The persistence of antibiotic resistance as a public health threat remains; and one way to help lessen this threat, is through the discovery of new, naturally-occurring, antimicrobials. Soil bacteria are known antimicrobial producers [4], and, indeed, a new class of antibiotics was recently discovered from soil [5].

This study identifies a strain of Pantoea annantis (MMB-1), isolated from soil, that demonstrates anti-microbial activity. In addition to observing this functional activity for MMB-1, the genome of MMB-1 was also sequenced. The genome of MMB-1 appears to contain genes believed to be involved in the production of secondary metabolites. Antibiotics are products of secondary, bacterial, metabolism [4]. Interestingly, strain MMB-1 is not the first $P$. annantis to show antimicrobial activity [1], thus, further highlighting the potential of this species as a source of antimicrobial substances. 


\section{Materials and Methods}

\section{Sample Collection, Strain Isolation and Strain Characterization}

The soil from which Pantoea ananatis MMB-1 was derived was originally collected in August 2017. Soil was collected $6 \mathrm{~cm}$ deep from the surface, in an area surrounding Franklin Cemetery, Northeast of Des Moines (IA, USA), at coordinates $41^{\circ} 42^{\prime} 53.4^{\prime \prime} \mathrm{N}$ and $93^{\circ} 22^{\prime} 46.6^{\prime \prime} \mathrm{W}$. The temperature of the soil at the time of collection was $23^{\circ} \mathrm{C}$ and the $\mathrm{pH}$ was 7.5.

One gram of collected soil was resuspended in 5 $\mathrm{mL}$ sterile water, homogenized by vortex, and serial-diluted (sterile water) for standard plate counting. Serial-diluted soil samples were plated on Potato Dextrose Agar (PDA) and incubated at $30^{\circ} \mathrm{C}$ for 4 days. Individual colonies were picked and streaked on PDA for isolation. Standard plate counting on PDA estimated the soil sampled contained $3.9 \times 10^{7} \mathrm{CFU} /$ gram of soil.

Basic morphological and biochemical tests were carried out against MMB-1, including Gram-staining, indole production, and catalase production, according to standard practice [6]. Isolated samples of MMB-1 were stored at $-80{ }^{\circ} \mathrm{C}$ for long-term use in Nutrient Broth (NB) supplemented with $10 \%$ (w/v), sterile, glycerol.

\section{Screening of MMB-1 for Antimicrobial Activity}

An antagonistic activity assay termed "spread-patch" [7], which is a form of competitive plating, was used to determine if MMB-1 can inhibit the growth of other bacteria when co-cultured on the same plate. Competitive plate can be used to screen bacteria for potential production of antimicrobial substances [7]. MMB-1 was screened for activity against a bacterium representing Gram-positives (Bacillus subtilis, ATCC 6051) and against a bacterium representing Gram-negatives (Klebsiella pneumoniae, ATCC 2342) using the procedure as described in [7]. In the procedure, a zone of no growth, around the plated "patch" of MMB-1, was interpreted as MMB-1 displaying antagonistic activity against the co-cultured bacterium.

\section{Crude Extraction of MMB-1 Antagonistic Substances}

Antagonistic activity identified by "spreadpatch" was confirmed as associated with MMB-1 via crude organic extraction from whole bacteria followed by testing of extracted materials for retained antagonistic activity against bacteria representing Gram-positives. This assay was carried out as described in [7], "Chemical Extraction of Antibiotics" section, using ethyl acetate as the extraction solvent.

\section{Genomic DNA Isolation}

Isolated MMB-1 was growth in $\mathrm{NB}$, and genomic DNA was isolated, according to the included instructions, using a PureLink Genomic DNA Mini Kit (Invitrogen). Integrity of isolated genomic DNA was visualized by agarose gel electrophoresis $(0.5 \%$ $\mathrm{w} / \mathrm{v})$. Isolated DNA was then provided to MR DNA (Shallowater, TX, USA) for whole genome sequencing and assembly.

\section{Bacterial Genome Sequencing}

Sequencing was performed using an Illumina HiSeq system. The concentration of DNA was evaluated using the Qubit ${ }^{\circledR}$ dsDNA HS Assay Kit (Life Technologies). The library was prepared using a Nextera DNA sample preparation kit (Illumina) following the manufacturer's user guide. Following library preparation, the final concentration of the library $(5.72 \mathrm{ng} / \mu \mathrm{L})$ was measured using the Qubit dsDNA HS assay kit (Life Technologies), and the average library size $(835 \mathrm{bp}$ ) was determined using the Agilent 2100 Bioanalyzer (Agilent Technologies). The libraries were pooled and diluted (to $6.0 \mathrm{pM}$ ) and sequenced paired end for 500 cycles (100X coverage). Obtained sequence reads were assembled (MR DNA) into 12 contigs (longest, 1,380,906 bp; N50, 664,687 bp) using NGen (DNASTAR). The resulting genome was annotated using RAST in default settings [8]. The software programs antiSMASH [9] and BAGEL [10] were used to identify potential secondary metabolite biosynthetic genes (most recent versions, default settings). This whole genome project was deposited at GenBank under accession (BioProject) PRJNA602096.

\section{Results and Discussion}

\section{Identification of MMB-1 from Soil}

The strain, designated here as Pantoea ananatis MMB-1 displayed characteristics shared among Pantoea including being found in soil, and composed of indole positive, Gram-negative rods, that displayed a mucoid-type morphology when grown on semisolid media (Figure 1). In addition, according to BLASTn analysis (NCBI, default parameters), a representative 16s rRNA gene from the sequenced genome of MMB-1 (Figure 4) showed $99.05 \%$ sequence identity to a $P$. ananatis $16 \mathrm{~s}$ rRNA gene in the NCBI database (strain PA13, accession CP003085.1). Consistent with BLASTn of 16s rRNA, BLASTp (NCBI, default parameters) using a common bacterial housekeeping gene, the chaperonin HSP60 (GroEL) protein, from the genome of MMB-1, showed $98.01 \%$ sequence identity to a $P$. ananatis GroEL gene in the NCBI database (strain LMG 20103, accession ADD75568). 

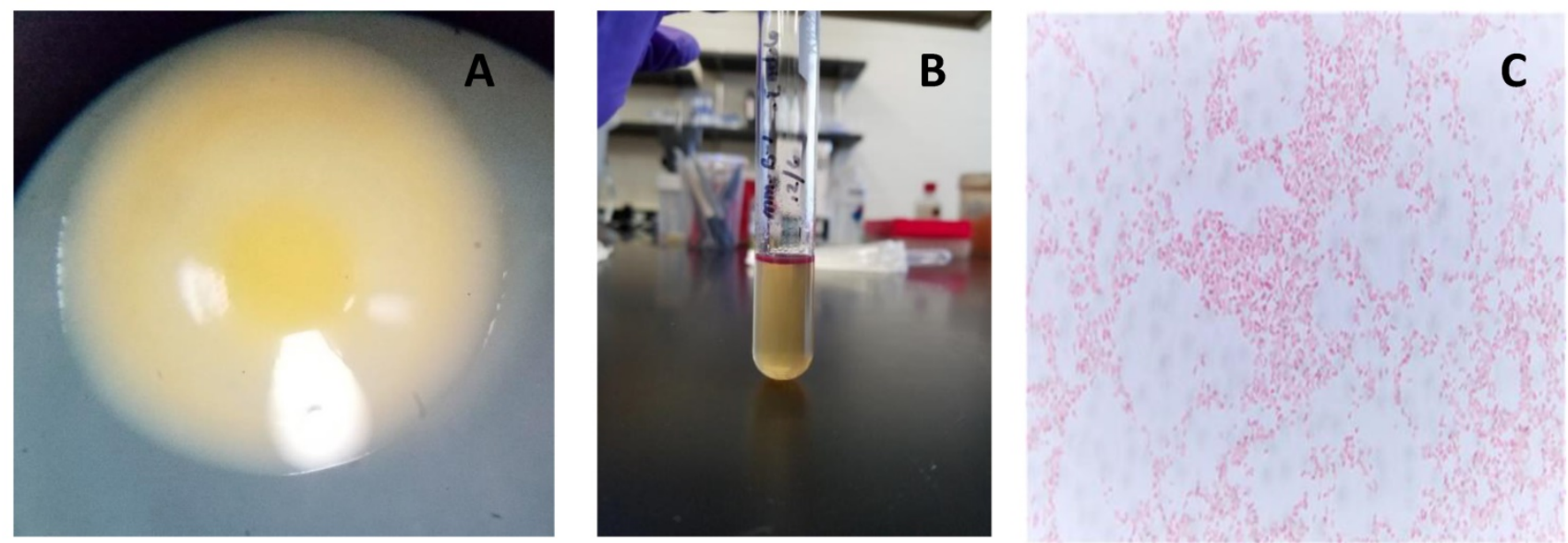

Figure 1. Observed characteristics of MMB-1. A. A representative colony of MMB-1 as observed at $40 \mathrm{X}$ using a stereomicroscope, showing yellow pigmentation, and a shiny, mucoid-type colony texture. B. A positive Indole test was observed (purple-pink color formed at top of test tube) after adding $0.5 \mathrm{~mL}$ of Kovac's reagent to MMB-1 grown at $37^{\circ} \mathrm{C}$ for 48 hours in $4.0 \mathrm{~mL}$ of tryptophan broth. C. A representative Gram-stained sample of MMB-1 as observed at $1,000 \mathrm{X}$ using brightfield light microscopy. Note the abundance of Gram-negative, single rods.
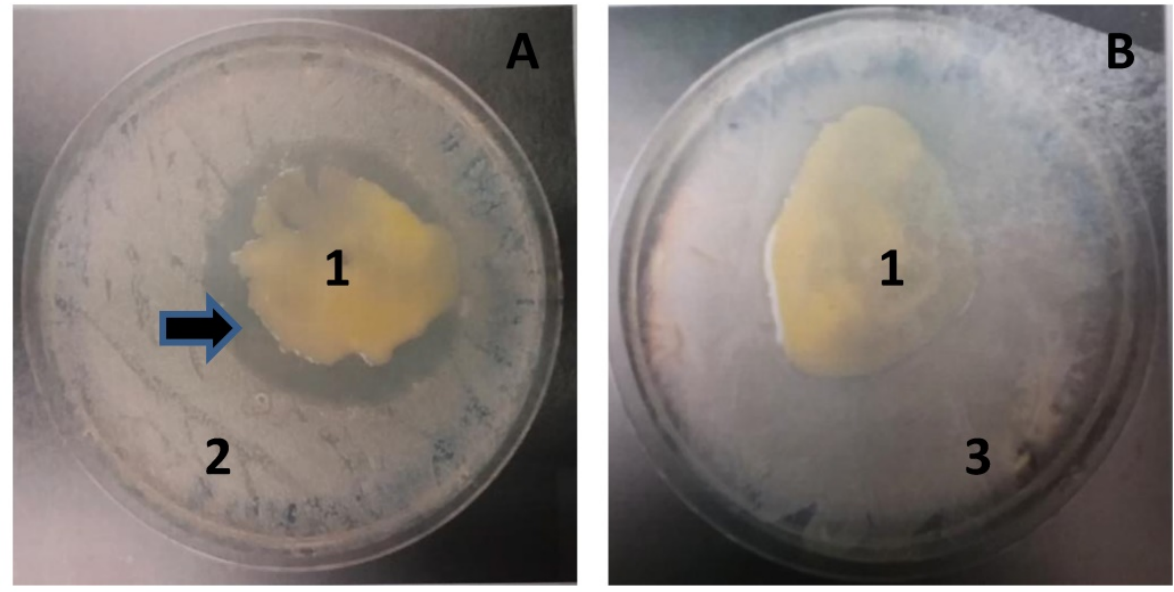

Figure 2. MMB-1 inhibits the growth of B. subtilis when co-cultured. A. Co-culture of MMB-1 (1) and B. subtilis (2). Note the zone of no growth surrounding MMB-1 (arrow). B. Co-culture of MMB-1 (1) and K. pneumoniae (3). Note the lack of zone of no growth in panel B. With both panel A and panel B, samples were growth for 48 hours at $37^{\circ} \mathrm{C}$ on PDA.

\section{MMB-1 Demonstrates Antimicrobial Activity}

Upon co-culturing MMB-1 with the Gram-positive bacterium B. subtilis, MMB-1 was observed to produce a zone of no growth surrounding the MMB-1 cells (Figure 2). This suggests that MMB-1 is producing a substance that is inhibiting the co-cultured B. subtilis. Such a zone of no growth was not observed when co-culturing MMB-1 with a Gram-negative bacterium, K. pneumoniae (Figure 2). Together, this indicates at least one antimicrobial substance is produced by MMB-1 that has potential to inhibit the growth of Gram-positives; but not Gramnegatives. Significantly, several antibiotic-resistant strains, Gram-positive in nature, are of current clinical concern [11].

To further confirm that MMB-1 is responsible for making a substance, or substances, that can account for the zone of no growth shown in Figure 2, an effort was made to extract organic substances from MMB-1.
Specifically, substances soluble in organic solvents (including ethyl acetate, used here), when extracted from whole bacterial cells, can often include antibiotics [7]. As shown in Figure 3, when ethyl acetate-based extracts of MMB-1 (from pure cultures of MMB-1) were exposed to B. subtilis, the extracts produce zones of no growth that are comparable to the live-organism-based experiment shown in Figure 2. Importantly, as also shown in Figure 3, exposure to the extraction solvent alone, ethyl acetate, alone does not inhibit the growth of $B$. subtilis.

\section{Genomic Features of MMB-1}

The draft genome of MMB-1 is 4,634,340 bp. The GC\% is $53.6 \%$. RAST (Rapid Annotation using Subsystem Technology) predicts a total of 4,624 genes. A genome map of MMB-1 is shown in Figure 4, as generated using the CGView server [12]. The genome size and GC\% values fall into ranges observed for other sequenced Pantoea, which appear to range from 
4.5 - 6.3 million bp in size and $52-55$ GC\% content [13]. The total number of genes encoded in the genome of MMB-1 is consistent with additional $P$. ananatis genomes sequenced [13]. As anticipated, a large number of subsystem feature counts identified by RAST are responsible for basic life-sustaining needs including 353 related to carbohydrate metabolism, 208 related to protein metabolism, 89 related to cellular respiration, 48 related to the bacterial cell wall, 46 related to membrane transport, and 44 related to signal transduction (Figure 5).
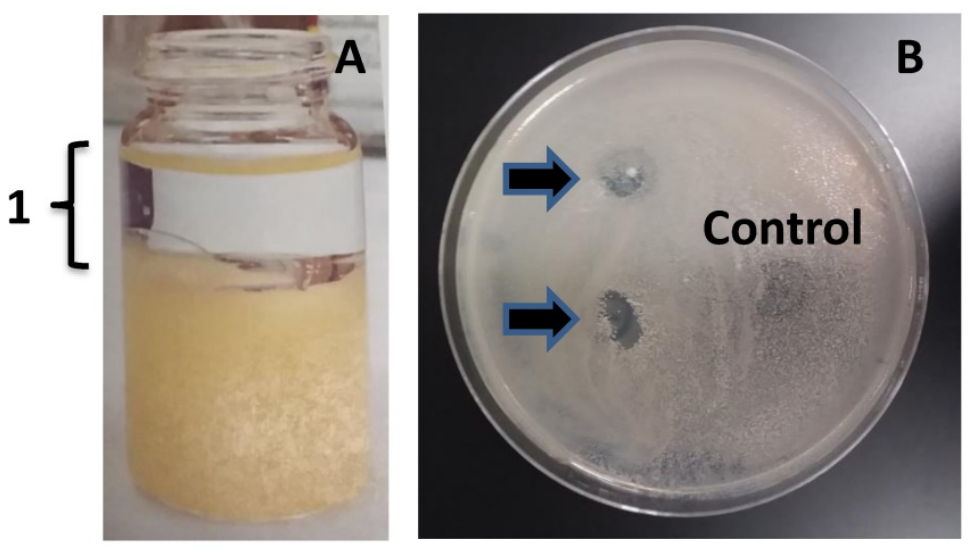

Figure 3. Ethyl acetate extract of MMB-1 retains the anti-microbial activity. A. Extract of MMB- 1 cells grown on PDA for 48 hours at $37^{\circ} \mathrm{C}(10 \mathrm{~mL}$ glass vial shown). In panel A, the organic phase of the extraction is indicated (1). B. Testing of organic phase (1, panel A). A total of $50 \mathrm{uL}$ (top arrow) and $100 \mathrm{uL}$ (bottom arrow) of organic phase extract were spotted on a PDA plate where a sample of $B$. subtilis was previously spread. Note the zones of no growth where organic extract was added (arrows). Control indicates where $100 \mathrm{uL}$ of ethyl acetate alone was added to the plate. Bacteria were allowed to grow for 48 hours at $37^{\circ} \mathrm{C}$ prior to documentation.

\section{Accession: PRJNA602096}

Length: $4,634,340 \mathrm{bp}$

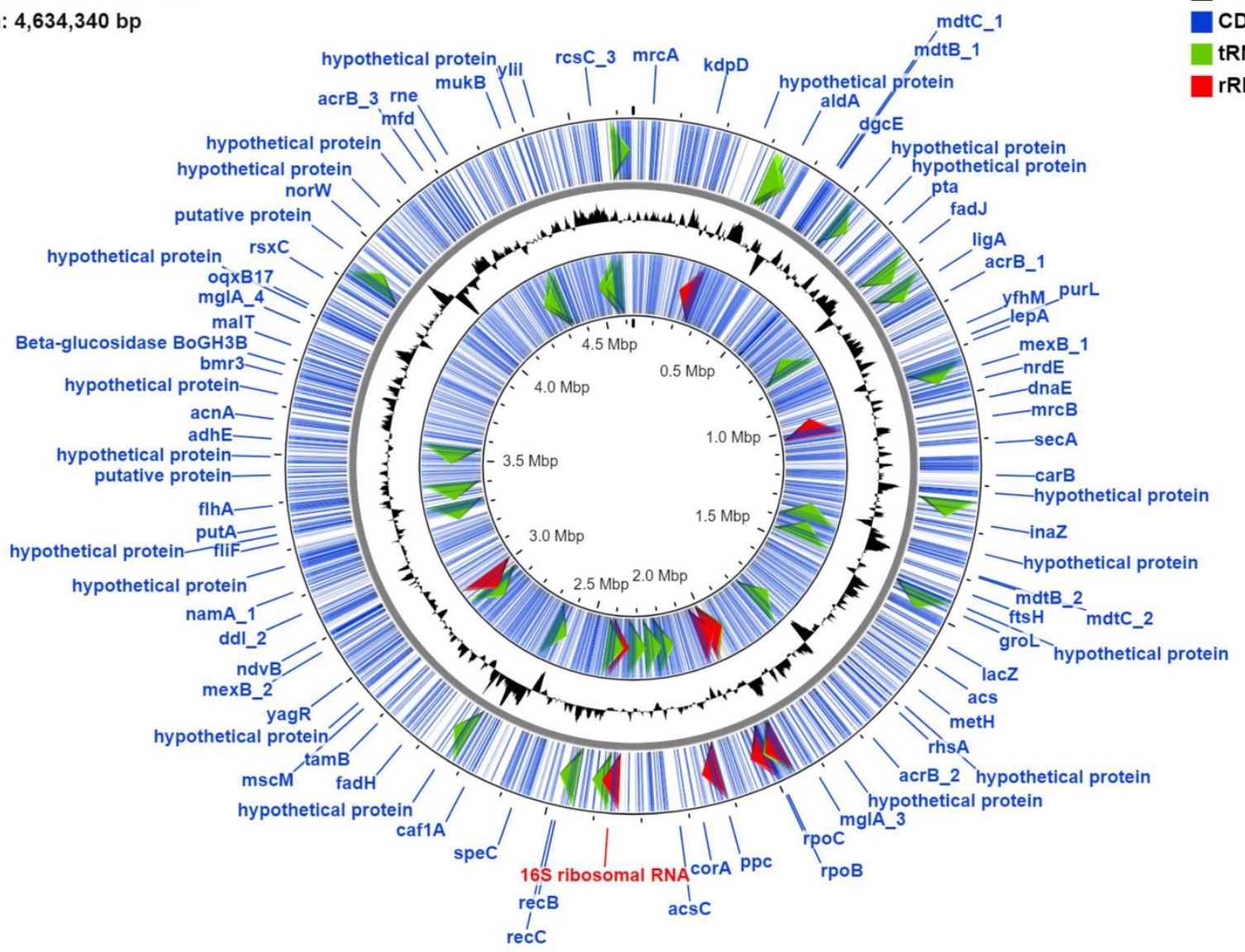

GC Content

CDS

TRNA

rRNA

Figure 4. Genome map of MMB-1. The map was generated using the default settings of CGView. Blue lines represent identified coding sequences (CDS), red arrows denote identified rRNA sequences, green arrows indicate tRNA sequences. Labels in blue (e.g. groEL, rpoB) are examples of gene names and their genomic locations. The signal graph in black mark GC\%. The inner ring labeled with multiple CDS is the reverse strand and the outer ring is the forward. The individual 165 rRNA marked red was used as the BLAST query sequence referenced in the results and discussion (Identification of MMB-1 from Soil). 


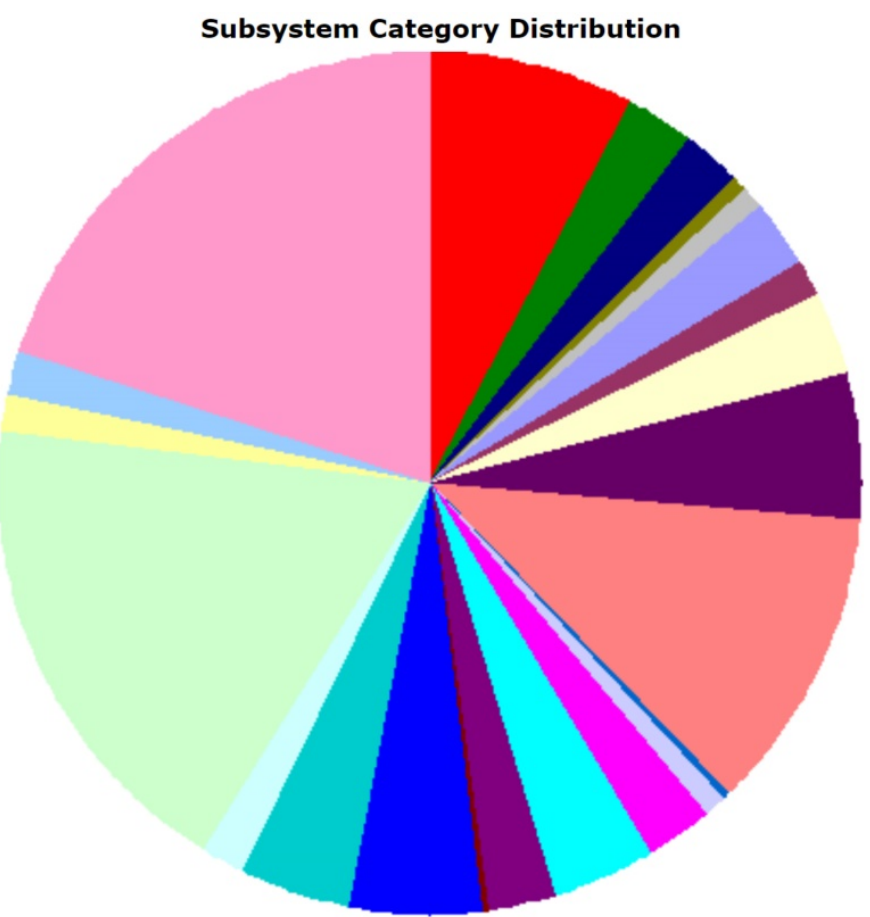

Subsystem Feature Counts

Ð Cofactors, Vitamins, Prosthetic Groups, Pigments (142)

$\boxplus$ Cell Wall and Capsule (48)

(1 Virulence, Disease and Defense (38)

$\boxplus$ Potassium metabolism (13)

$\boxplus$ Photosynthesis (0)

$\boxplus$ Miscellaneous (12)

$\boxplus$ Phages, Prophages, Transposable elements, Plasmids (0)

$\boxplus$ Membrane Transport (46)

$\boxplus \square$ Iron acquisition and metabolism (28)

$\boxplus \quad$ RNA Metabolism (54)

$\boxplus \square$ Nucleosides and Nucleotides (100)

$\boxplus$ Protein Metabolism (208)

$\boxplus \square$ Cell Division and Cell Cycle (7)

Ð- Motility and Chemotaxis (14)

$\boxplus \square$ Regulation and Cell signaling (44)

Ð Secondary Metabolism (5)

$\boxplus$ DNA Metabolism (71)

$\boxplus$ Fatty Acids, Lipids, and Isoprenoids (47)

$\boxplus$ Nitrogen Metabolism (7)

$\boxplus$ Dormancy and Sporulation (1)

Respiration (89)

$\boxplus$ - Stress Response (77)

$\boxplus \quad$ Metabolism of Aromatic Compounds (27)

$\boxplus \quad$ Amino Acids and Derivatives (332)

$\boxplus$ Sulfur Metabolism (26)

$\boxplus$ Phosphorus Metabolism (31)

$\boxplus$ Carbohydrates (353)

Figure 5. Subsystem distribution of the MMB-1 genome. Category distribution is shown, as outputted from RAST. Feature counts the predicted number of genes found by RAST that belong to each functional category. A total of 1,272 genes ( $28 \%$ of total annotated genes) could be placed, stringently, into RAST-based feature count categories, while 3,352 could not.

\subsection{5.contigs.fa AOI_01}

Gene names

Predicted promoters

Predicted terminators

Show or hide small ORFS

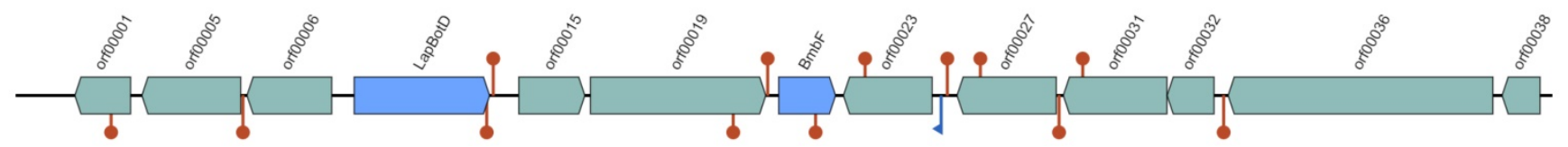

Figure 6. MMB-1 genome analysis by BAGEL. According to BAGEL software, the genome of MMB-1 contains a gene cluster with potential to synthesize the bacteriocin (antimicrobial peptide) bottromycin. The graphic (screenshot) shows the organization of genes within this cluster, relative to one another. The genes shaded in blue are locations of genes specifically-linked to bottromycin, BmbF and LapBotD.

Given the potential anti-microbial activity observed by MMB-1 (Figures 2 and 3), an effort was made to identify potential genes that might contribute to such biochemical activity. BAGEL is a web-based server that identifies potential bacteriocin open reading frames from inputted sequences, via the querying of knowledge-based bacteriocin databases [10]. Bacteriocins are a diverse group of antimicrobial peptides produced by bacteria [14]. According to BAGEL, the MMB-1 genome contains a set of genes that share similarity in sequence, and on the basis of proximity of one another, to a known bacteriocin biosynthetic pathway (Figure 6). Of particular note, this segment of genes (Figure 6), according to BAGEL, contains those related to $\mathrm{BmbF}$ and LapBotD (YcaO), both of which are believed to play a role in the production of the bacteriocin bottromycin [15].
Interestingly, and consistent with our biochemical data (Figures 2 and 3), bottromycin and bottromycinlike antibiotic peptides appear to show significant antimicrobial activity against Gram-positives [16]; although, some also appear to have activity against Gram-negatives.

The genome of MMB-1 was also examined using antiSMASH. This software is a pipeline for secondary metabolite gene cluster identification. It looks at a variety of secondary metabolism-related genes including non-ribosomal peptides, lantibiotics, and siderophores [9]. According to antiSMASH, the genome of MMB- 1 contains 23 candidate regions. This value is consistent when compared to other Pantoea genomes that have been probed for secondary metabolite gene clusters using antiSMASH; with the range being from 16 to 28 clusters per genome [13, 17]. 
Query sequence

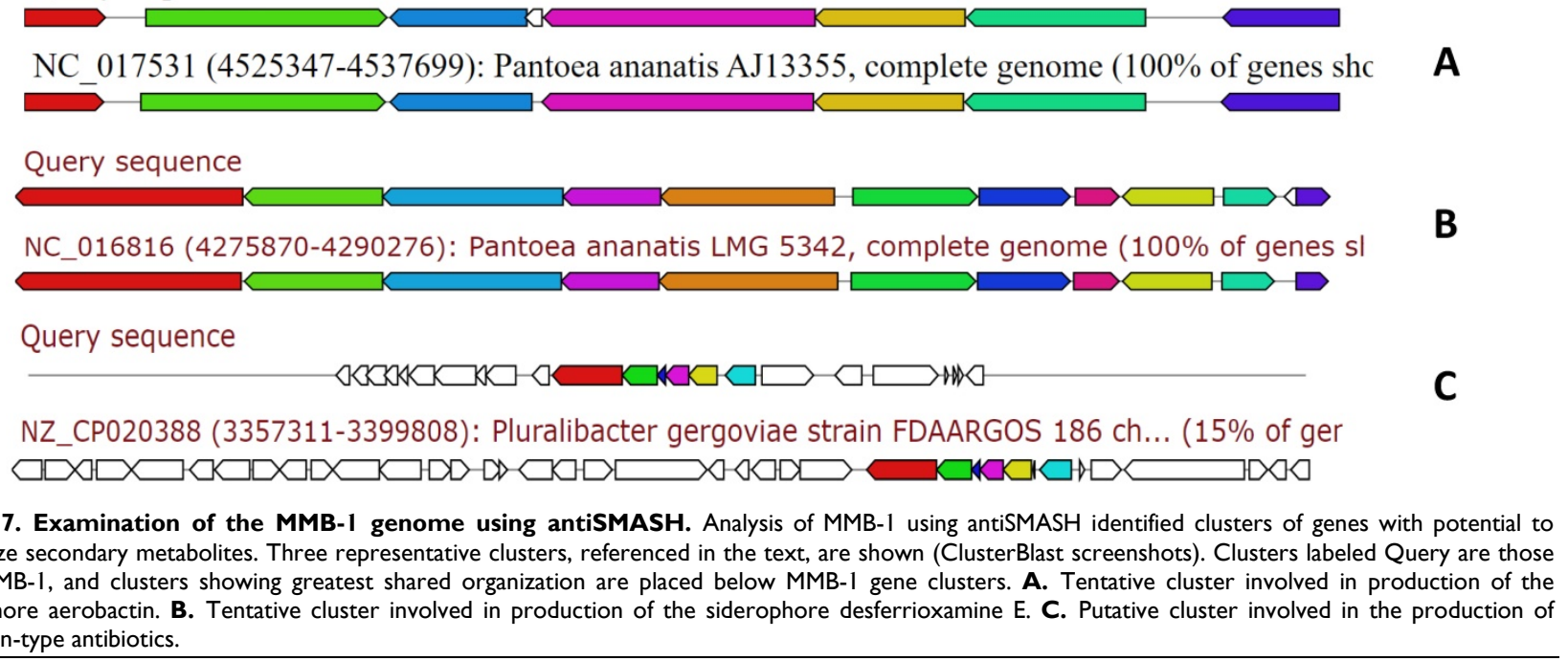

Also consistent with other Pantoea genomes scanned by antiSMASH, MMB-1 contains putative biosynthetic gene clusters for the siderophores aerobactin and desferrioxamine $\mathrm{E}$, as well as a tentative cluster for the exopolysaccharide stewartan $[13,17]$. Interestingly, antiSMASH also identified a cluster for lankacidin C (Figure 7). Lankacidin-type antibiotics are products first identified in soildwelling Streptomyces, and which show significant antimicrobial activity against Gram-positive bacteria $[18,19]$. In comparison to 31 Pantoea genomes recently scanned by BAGEL and antiSMASH, MMB-1 appears unique in generating hits for lankacidin $\mathrm{C}$ or bottromycin $[15,20]$.

In summary, the study here identified a strain of Pantoea ananatis (MMB-1) from soil, that appears capable of producing at least one antimicrobial substance, according to collected phenotypic and genotypic data. These findings increase our overall understanding of Panteoa as a group, and are also consistent with the notion that members of this group have significant potential as useful natural product producers, including as yet to be discovered antimicrobials. Future studies will seek to isolate and structurally-characterize anti-microbial products synthesized by MMB-1.

\section{Acknowledgements}

We thank all members of the Biology Department at Grand View University for encouragement and project support. The authors do not have any conflict of interest to declare. We also thank Jennifer Donnelly for technical support, Dr. Scot Dowd (MR DNA) for genome sequencing support, and the Tiny Earth initiative for project inspiration.

\section{Competing Interests}

The authors have declared that no competing interest exists.

\section{References}

1. Walterson AM, Stavrinides J. Pantoea: insights into a highly versatile and diverse genus within the Enterobacteriaceae. FEMS Microbiol Rev. 2015; 39: 968-84.

2. Pusey PL, Rudell DR, Stockwell VO. Mechanisms of Pantoea agglomerans strain e325 as antagonist of Erwinia amylovora. Acta Hortic. 2008; 457-60.

3. Audu KE, Adeniji SE, Obidah JS. Bioremediation of toxic metals in mining site of Zamfara metropolis using resident bacteria (Pantoea agglomerans): a optimization approach. Heliyon. 2020; 6: e04704.

4. Mohr KI. History of Antibiotics Research. Curr Top Microbiol Immunol. 2016; 398: 237-272.

5. Hover BM, Kim S-H, Katz M, et al. Culture-independent discovery of the malacidins as calcium-dependent antibiotics with activity against multidrug-resistant Gram-positive pathogens. Nat Microbiol. 2018; 3: 415-22.

6. Smith H, Brown AE. Benson's microbiological applications: laboratory manual. Fifteenth edition. New York: McGraw Hill; 2022.

7. Hernandez S, Tsang T, Bascom-Slack C, Broderick N, Handelsman J. Tiny earth, a resource guide to student sourcing antibiotic discovery. Massachusetts: XanEdu; 2018.

8. Aziz RK, Bartels D, Best AA, et al. The RAST server: rapid annotations using subsystems technology. BMC Genomics. 2008; 9: 75.

9. Blin K, Shaw S, Kloosterman AM, et al. antiSMASH 6.0: improving cluster detection and comparison capabilities. Nucleic Acids Res. 2021; 49: W29-35.

10. de Jong A, van Hijum SA, Bijlsma JJ, Kok J, Kuipers OP. BAGEL: a web-based bacteriocin genome mining tool. Nucleic Acids Res. 2006; 34: W273-9.

11. Mulani MS, Kamble EE, Kumkar SN, Tawre MS, Pardesi KR. Emerging strategies to combat ESKAPE pathogens in the era of antimicrobial resistance: a review. Front Microbiol. 2019; 10: 539.

12. Stothard P, Grant JR, Van Domselaar G. Visualizing and comparing circular genomes using the CGView family of tools. Brief Bioinform. 2019; 20: 1576-82.

13. Weller-Stuart T, De Maayer P, Coutinho T. Pantoea ananatis: genomic insights into a versatile pathogen. Mol Plant Pathol. 2017; 18: 1191-8.

14. Soltani S, Hammami R, Cotter PD, et al. Bacteriocins as a new generation of antimicrobials: toxicity aspects and regulations. FEMS Microbiol Rev. 2021; 45: fuaa039.

15. Williams AN, Sorout N, Cameron AJ, Stavrinides J. The integration of genome mining, comparative genomics, and functional genetics for biosynthetic gene cluster identification. Front Genet. 2020; 11: 600116.

16. Franz L, Kazmaier U, Truman AW, Koehnke J. Bottromycins biosynthesis, synthesis and activity. Nat Prod Rep. 2021; 38: 1659-83. 
17. Hara $\mathrm{Y}$, Kadotani N, Izui $\mathrm{H}$, et al. The complete genome sequence of Pantoea ananatis AJ13355, an organism with great biotechnological potential. Appl Microbiol Biotechnol. 2012; 93: 331-41.

18. Ayoub AT, Abou El-Magd RM, Xiao J, et al. Antitumor activity of lankacidin group antibiotics is due to microtubule stabilization via a paclitaxel-like mechanism. J Med Chem. 2016; 59: 9532-40.

19. Auerbach T, Mermershtain I, Davidovich $C$, et al. The structure of ribosome-lankacidin complex reveals ribosomal sites for synergistic antibiotics. Proc Natl Acad Sci. 2010; 107: 1983-8.

20. Lee SI, Tran TD, Huynh S, Parker CT, Hnasko R, McGarvey JA.

Complete genome sequence of Pantoea agglomerans ASB05 using Illumina and PacBio sequencing. Microbiol Resour Announc 2021; 10: e00501-21. 